\title{
DETERMINACIÓN DE TRAYECTORIAS DE BLOQUES ROCOSOS EN ENSAYOS MEDIANTE VIDEOGRAMETRÍA
}

\author{
DETERMINATION OF ROCK BLOCK TRAJECTORIES IN DROP TESTS USING VIDEOGRAMMETRY

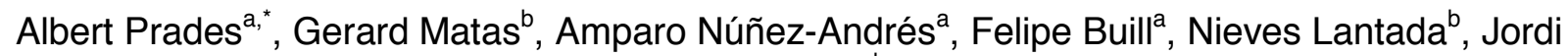 \\ Corominas $^{b}$ \\ ${ }^{a}$ Departamento de Ingeniería Civil y Ambiental, Escuela Politécnica Superior de Edificación de Cataluña, Universitat Politècnica de \\ Catalunya, Avda. Dr. Marañón 44-50, 08028, Barcelona. alberto.prades.i@upc.edu \\ ${ }^{\mathrm{b}}$ Departamento de Ingeniería Civil y Ambiental, Escuela Técnica Superior de Ingeniería de Caminos, Canales y Puertos de Barcelona, \\ Universitat Politècnica de Catalunya, C/ Jordi Girona 1-3, 08034, Barcelona.
}

\begin{abstract}
:
Determining the trajectory of a block that falls down on a slope is not straightforward due to the high number of variables involved. Because of this, the appropriate understanding of how these variables may affect the trajectories is required.

In this work, we estimate some magnitudes that affect the trajectories of the blocks such as the coefficient of restitution and the energy thresholds for fragmentation of the blocks among others. We have chosen the controlled scenario of a quarry in which targets and high speed video cameras were arranged to capture images of the blocks falling from the top of a slope. The position of the blocks over time has been measured from video frames applying photogrammetric methods. From here, the velocities and the accelerations have been determined, and with the estimated mass of each block, we have calculated the energy balance.
\end{abstract}

Key words: Geomatics, Photogrammetry, Videogrammetry, 3D Models, Rock falls.

\section{Resumen:}

La trayectoria de un bloque que cae por un talud no es fácil de determinar porque influyen muchas variables difíciles de cuantificar. Ello requiere del conocimiento de cómo estas variables afectan a las trayectorias.

En este trabajo se estiman algunas magnitudes que afectan la trayectoria de los bloques como son los coeficientes de restitución y los límites de energía para generar la fragmentación del bloque, entre otros. Para ello se ha escogido el entorno controlado de una cantera, en el que se han dispuesto dianas y cámaras de vídeo de alta velocidad, para capturar imágenes de las caídas de bloques lanzados desde lo alto de un talud. Hemos medido la posición del bloque respecto del tiempo a partir de los fotogramas de vídeo obtenidos, aplicando métodos fotogramétricos. A partir de aquí se determinan las velocidades y las aceleraciones y, con la estimación de la masa de cada bloque, hemos calculado los balances de energía.

Palabras clave: Geomática, Fotogrametría, Videogrametría, Modelos 3D, Desprendimientos rocosos.

\section{Introducción}

Se entiende por desprendimientos rocosos las masas de roca que, por algún motivo, se separan de una pared y se propagan por la pendiente en caída libre, rebotando, rodando y fragmentándose. Los desprendimientos rocosos pueden ocurrir en cualquier instante indeterminado $y$ en cualquier lugar del territorio susceptible de este tipo de sucesos, especialmente en zonas montañosas de topografía accidentada o en lugares próximos a acantilados. También se caracterizan por ser fenómenos extraordinariamente rápidos de los cuales cuando se producen, no hay posibilidad de escapar. En estas condiciones, el riesgo que se produzcan víctimas o desperfectos en edificios e infraestructuras es altamente probable y la única solución posible es la prevención tomando las medidas de protección oportunas (Corominas and Mavrouli 2011; Corominas et al. 2013).

La prevención es posible si, en las zonas de peligrosidad, se tiene conocimiento a priori de los volúmenes movilizables, de las posibles trayectorias que los bloques pudieran trazar durante su caída y de las energías transportadas por dichos bloques o sus fragmentos en el caso de que se produzcan roturas.

Durante los últimos cuatro años nuestro grupo de investigación ha desarrollado modelos que simulan desprendimientos rocosos. Nuestro objetivo es la prevención de este tipo de sucesos en las zonas de riesgo, proporcionando criterios para el diseño de sistemas de protección. Información adicional sobre nuestros trabajos de investigación se puede encontrar en: https://rockmodels.upc.edu/es

‘Corresponding Author: Albert Prades, alberto.prades.i@upc.edu 
Los modelos de fragmentación y propagación de los desprendimientos requieren conocer el comportamiento de un bloque rocoso en su caída por una ladera. Con este propósito, hemos realizado diversos ensayos de caída controlada de bloques de distintos tamaños y características. Su trayectoria de caída se ha registrado mediante cámaras de vídeo de alta velocidad. El interés lo centramos en la extracción de datos fundamentales que contesten preguntas sobre la propagación de un bloque: ¿con qué frecuencia se da la fragmentación?, ¿existen límites de energía que garanticen que un bloque se va a fragmentar?, ¿qué valores medios tienen los coeficientes de restitución en un substrato determinado?, ¿merece la pena incluir la energía de rotación de los bloques en nuestros modelos o puede ser menospreciada?, etc.

Se considera que desde el momento en que se ha producido la rotura de la pared rocosa, la propagación del desprendimiento está condicionada por las dimensiones de la masa separada, la geometría de la vertiente, las propiedades elásticas del terreno y la presencia de obstáculos. El análisis de la propagación de los desprendimientos es fundamental para cuantificar el riesgo (Volkwein et al. 2011) porque define las trayectorias futuras de los bloques rocosos, la altura de los rebotes que se puedan producir, las velocidades y los puntos de impacto. El conocimiento preciso de estos parámetros permitirá determinar la probabilidad de impacto sobre los elementos expuestos, su vulnerabilidad y el diseño de los elementos de protección que se desee instalar.

La capacidad de destrucción de un desprendimiento viene dada por la energía cinética del bloque. En un desprendimiento, los bloques se propagan con distintas velocidades $y$, por lo tanto, es necesario ver la intensidad del impacto como un parámetro espacialmente distribuido (Fig. 1).

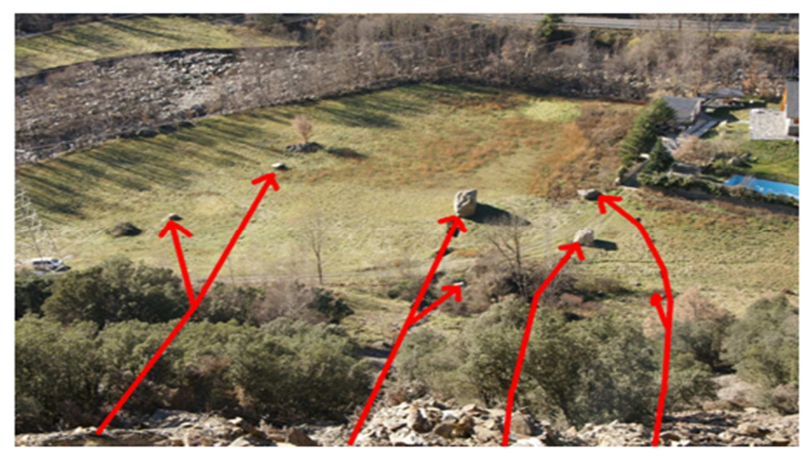

Figura 1: Desprendimiento rocoso en el municipio de Escaló (Lleida). En rojo se muestran las trayectorias seguidas por cada bloque.

Hay grupos de trabajo que están implementando modelos numéricos, tanto bidimensionales como tridimensionales, con los que pretenden calcular precisamente la distribución de las energías de impacto para un hipotético desprendimiento. Así tenemos los programas: ROCFALL (Stevens 1998), el Colorado Rockfall Simulation Program (CRSP) (Jones et al. 2000), HY-STONE (Crosta and Agliardi 2003), Rockyfor 3D (Dorren et al. 2010) o el que se está desarrollando actualmente en nuestro grupo de investigación, llamado RockGIS (Matas et al. 2017a; Matas et al. 2017b). Estos modelos permiten generar mapas temáticos donde se muestra las trayectorias más probables, las alturas de rebote y la distribución de energías de impacto para cada unidad espacial o celda. La información recogida permite, en un paso posterior, la determinación de la probabilidad de impacto contra los elementos expuestos y la cuantía de los daños esperables en cada caso. Estos mapas son ayudas valiosas para el diseño de los sistemas de protección (barreras dinámicas, terraplenes de protección, etc.), para establecer cuáles son los requisitos mínimos que deben cumplir y cuál es la mejor situación para instalarlos.

En su publicación, Li and Lan (2015) hacen una revisión de las incertidumbres de los modelos de simulación de trayectorias de bloques y remarcan que, al menos hasta entonces, el único modelo que incluye la fragmentación es el programa HY-STONE. En el modelo de desprendimientos que actualmente desarrolla nuestro grupo se hace hincapié en la importancia de considerar la fractura del bloque que está cayendo. Sabemos que el mecanismo de fragmentación de una masa rocosa no es trivial (Zang et al. 2000; Chau et al. 2002) y que son diversos los parámetros que afectan la fragmentación de un bloque (Wang and Tonon 2011): la presencia de discontinuidades, incluyendo la persistencia y la orientación, la energía del impacto, la rigidez del terreno y el ángulo de incidencia del impacto afectan el modo de disgregación en fragmentos. A su vez e independientemente, cada fragmento seguirá cayendo, rodando y probablemente fragmentándose de nuevo.

Giacomini et al. (2009; 2012) hicieron ensayos de caída de bloques en una cantera pero no pudieron definir claramente el umbral de energía de impacto que determinara la fragmentación de un bloque. Se han probado, también, modelos numéricos de elementos discretos para modelizar la fragmentación y los resultados muestran que la fragmentación condiciona la extensión del depósito de bloques (Salciarini et al. 2009; Wang and Tonon 2011).

A pesar de los esfuerzos que se han hecho hasta la fecha, la modelización física del proceso de fragmentación aún está lejos de estar resuelta. Por este motivo, Matas et al. (2017) han desarrollado un programa de simulación de trayectorias de bloques que incorpora un módulo de fractura de base estocástica, para determinar si un bloque se fragmenta o no en el momento del impacto. La regla para decidir si un bloque se rompe debe ser compatible con las observaciones empíricas de las caídas de bloques hechas en ensayos en las canteras Foj (campaña 2015) y La Ponderosa (campaña 2016), auspiciadas por el proyecto Rockrisk (Corominas et al. 2017).

Es fundamental también considerar la transmisión de la energía del bloque inicial a los fragmentos. En nuestro modelo, al producirse un impacto, parte de la energía cinética es disipada por la deformación del suelo y parte por la deformación o fragmentación del bloque. Una parte de esta energía se la lleva el bloque que rebota o los fragmentos (si el bloque se rompe) dispersados. En el momento del impacto puede ocurrir que una parte de la energía cinética inicial pase a energía de rotación de los bloques. Una manera empírica para establecer los valores de los parámetros de restitución es estudiar en detalle el impacto de un bloque real. 


\section{Objetivos}

El objetivo de este trabajo consiste en analizar la caída de un bloque en un entorno controlado. Mediante métodos fotogramétricos se pretende medir de manera indirecta y precisa las posiciones, velocidades y aceleraciones del bloque a lo largo de su trayectoria. Con la estimación de la masa, asumiendo una densidad conocida, se calculan las energías involucradas en los instantes previos y posteriores a los impactos y a partir de aquí se estima la energía disipada durante el impacto. Consideraremos que la energía disipada se reparte entre la deformación del terreno que soporta el impacto y la deformación del propio bloque.

También nos proponemos medir la velocidad de rotación del bloque y cómo varía a causa de los sucesivos impactos contra la superficie del talud. Se analiza la transferencia de energía rotacional, que no es tenida en cuenta en la mayoría de modelos, y estudiamos si merece la pena incorporarla en el análisis de propagación. Algunas trayectorias de fragmentos de pequeño tamaño, observadas en las imágenes de los vídeos, nos hacen pensar que la respuesta es afirmativa. Creemos que, en algunos casos, un bloque que impacta contra el talud puede aumentar considerablemente la velocidad de rotación. Si en esas condiciones se desprende un pequeño fragmento del bloque en el instante adecuado, podría adquirir una velocidad ascendente suficiente para sobrepasar cualquier obra de defensa (por ejemplo, las barreras de seguridad instaladas en ese punto).

Finalmente, en el caso de que se produzca la fragmentación del bloque realizamos el seguimiento de, al menos, los fragmentos más grandes o de aquellos que, aún siendo pequeños, pudieran tener interés, por ejemplo, porque hayan sido eyectados a gran velocidad. La medida precisa de la posición de los fragmentos dará pistas tales como la distribución de sus velocidades después del rebote y se podrá determinar si los fragmentos quedan confinados dentro de un cono de eyección. En la medida que se estimen las masas de los fragmentos se podría estudiar el balance de energías en el proceso de impacto con fragmentación.

\section{Campaña de campo}

Durante los días 17 y 18 de junio de 2015 se realizaron dos ensayos de caída de bloques a escala real en la cantera Foj situada en Vallirana (Barcelona). La altura total, teniendo en cuenta la altura de la maquinaria empleada para lanzar los bloques, va desde los $16.5 \mathrm{~m}$ hasta los $27.5 \mathrm{~m}$. Se lanzaron un total de 56 bloques con volúmenes comprendidos entre 0.2 y $4.8 \mathrm{~m}^{3}$ (Gili et al. 2016). Antes de empezar el ensayo se hizo una cobertura fotográfica alrededor de cada bloque de manera que se pudiera, posteriormente, construir un modelo tridimensional detallado para cada uno de ellos. Paralelamente, en las inmediaciones de la zona se instalaron 12 dianas fijas que hacían las veces de marco sobre el que referir todas las medidas de posición. Las coordenadas locales de los centros de las dianas se midieron cuidadosamente dos o tres veces, a lo largo del tiempo que duraron los ensayos, con una estación total Leica TS06. El levantamiento estaba apoyado por dos receptores GPS Leica serie 530 que nos permitieron pasar las coordenadas locales a sus correspondientes proyecciones en UTM 31N (ETRS89) y cota ortométrica (modelo de geoide EGM08D595), que es el sistema de coordenadas oficial.

Los ensayos fueron grabados con tres cámaras Sony NEX-FS700R de alta velocidad y alta definición situadas estratégicamente para que nos permitiera triangular en una fase posterior. Las dianas se usaron como puntos de apoyo para el bloque fotogramétrico formado por las tres imágenes obtenidas por las cámaras de vídeo.

Por otra parte, se hicieron tomas de vídeo convencional de cada evento con una cámara GoPro Hero 4 a título de documentación del experimento. $Y$ una toma fotográfica de la berma con una cámara Nikon 5300 con objetivo de $18 \mathrm{~mm}$ de distancia focal a una resolución de $6000 \times 4000$ píxeles donde se hizo el ensayo, que nos permitió hacer un levantamiento fotogramétrico del que se obtuvo un modelo tridimensional de la zona.

\section{Procesamiento de las imágenes de vídeo}

El levantamiento topográfico de las dianas nos da el marco de referencia espacial y la base de tiempo de las cámaras de vídeo (1/400 s) nos da el marco temporal. Este montaje nos permite medir las posiciones de un objeto móvil respecto del tiempo y, por lo tanto, su velocidad y aceleración.

\subsection{Sincronía entre las tomas de un bloque}

En el mercado de instrumentación fotográfica se pueden encontrar artilugios de distinta índole para disparar dos o más cámaras simultáneamente pero, en general, su alcance está restringido a unas distancias de unos pocos metros. En nuestro caso, las cámaras de vídeo están separadas por unos 50 o $60 \mathrm{~m}$ entre sí y, en consecuencia, fuera del alcance del disparador. Para evitar complicaciones y encarecer el circuito de disparo se optó por hacer uso de un flash visible por las tres cámaras que, activado en algunos instantes oportunos (al principio, hacia la mitad y al final del lanzamiento), servirían de señal de sincronía.

En el programa que estamos desarrollando hemos añadido un módulo que busca los destellos del flash entre los fotogramas de cada vídeo. Para cada señal que encuentra (suelen ser 2 o 3 ) deja un registro del fotograma donde ha sucedido el destello. Al acabar el proceso de detección se tienen dos o tres marcas de sincronía para cada toma. A partir de aquí, el programa permite al usuario asignar manualmente las correspondencias de los destellos entre vídeos. Una vez se haya acabado este proceso se dispondrá de las tres tomas de vídeos sincronizadas.

Uno de los problemas que hemos detectado es que la velocidad de obturación de las cámaras de vídeo es tan rápida que, en algunos casos, el destello de luz se produce entre dos fotogramas consecutivos y no queda registrado. En estos casos no queda más remedio que encontrar la sincronía a mano; por ejemplo, observando la zona más baja de la trayectoria del bloque, donde su velocidad es máxima, y decidir a criterio del observador el instante del impacto. Es decir, registramos el fotograma anterior al del impacto como marca de sincronía. Por fortuna, del centenar de bloques lanzados sólo ha ocurrido en unos pocos casos y el inconveniente 
principal es que hay que dedicar un poco más de tiempo en encontrar la sincronía entre los vídeos.

\subsection{Extracción de la posición de un bloque por fotogrametría}

El paso siguiente consiste en relacionar las coordenadas imagen de las dianas medidas sobre cada fotograma, a través de las ecuaciones de proyección central, con las correspondientes coordenadas terreno (Hartley and Zisserman 2008). Con el número suficiente de puntos de los que se han medido las coordenadas imagen y las coordenadas terreno se puede estimar la posición del centro de proyección y la orientación que tenía la cámara en el momento que se tomó aquel fotograma. Este proceso es conocido como orientación externa y representa la reconstrucción de la posición en el espacio del haz de rayos de luz que generaron la imagen.

Una vez se dispone de la orientación externa de las tres cámaras se puede, para cualquier objeto medido sobre, al menos, dos imágenes, por intersección de haces de luz, las correspondientes coordenadas terreno. Nuestro objeto de interés es el bloque que cae por la pendiente, como se muestra en la Figura 2.

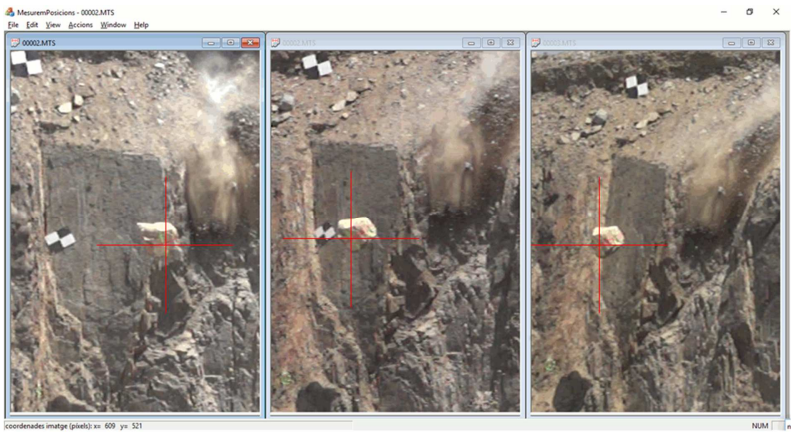

Figura 2: Una muestra de nuestro programa en el que vemos la medida simultánea de un punto sobre el bloque desde tres puntos de vista distintos.

Se puede ver que la iluminación, el contraste, las sombras, la baja resolución espacial y la forma no geométrica del objeto hacen difícil encontrar puntos homólogos de calidad entre los fotogramas. Además, sólo se puede medir sobre la superficie del bloque y no se sabe a priori donde queda proyectado el centro de masas, que sería el verdadero punto de interés a medir.

\subsection{Modelo de los bloques}

Durante la campaña de campo se tomó un juego de fotografías alrededor de cada bloque antes de lanzarlos, lo que permitió hacer un modelo tridimensional de cada uno de ellos con la ayuda del programa comercial Agisoft PhotoScan. Hemos desarrollado un módulo en nuestro programa que, partiendo del modelo del bloque, calcula la posición del centro de masas y lo muestra proyectado sobre la superficie del modelo. Nuestra idea es que, por comparación entre el modelo y las respectivas imágenes de vídeo, se sabe en qué punto del bloque se ha de capturar la coordenada imagen apropiada. En la Figura 3 se muestran algunos ejemplos en los que se ha modificado el valor de la transparencia del modelo de modo que puede verse en el interior el centro de masas y los ejes principales del bloque.

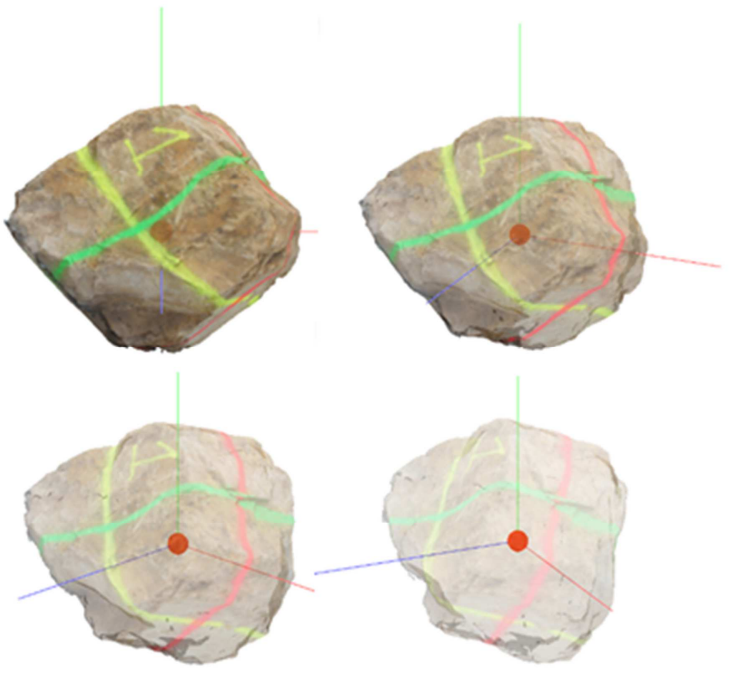

Figura 3: Una muestra de nuestro programa en el que vemos la medida simultánea de un punto sobre el bloque desde cuatro puntos de vista distintos. El programa permite ajustar la transparencia del bloque y permite ver, en el interior, el centro de masas y los ejes principales.

Los bloques lanzados en este estudio en particular son graníticos con una densidad media de $2700 \mathrm{~kg} / \mathrm{m}^{3}$. El modelo a escala de cada bloque nos permite hacer el cálculo del volumen y con la densidad propuesta se obtiene la masa.

Como se ha dicho en el apartado 2 está previsto que en este trabajo se haga el estudio del efecto de la energía de rotación, por lo tanto, hace falta calcular previamente el tensor de inercia de cada bloque que viene dado por la expresión, Ec. (1):

$$
I=\int_{V} r^{2} d m
$$

El cálculo del tensor de inercia depende del sistema respecto del que están referidos los puntos de la nube del modelo del bloque. Este sistema de referencia es arbitrario y lo define el levantamiento fotogramétrico de cada bloque; a los que sólo se han dotado de escala. Así pues, el paso siguiente es calcular el centro de masas del bloque y situar el origen del sistema de referencia sobre él. Posteriormente, se diagonaliza la matriz del tensor de inercia con lo que quedan determinados los tres ejes principales que servirán, a partir de aquí, de sistema de referencia local para cada bloque. En nuestro caso, para calcular los valores y vectores propios hemos usado el método de Jacobi que implementa las librerías de Numerical Recipes for C++ (Press et al. 2007).

En el programa que estamos desarrollando hemos incorporado un método para calcular el tensor de inercia de un sólido a partir de la nube de puntos de su superficie (Blow and Binstock 2004), que evita la integración numérica directa sobre el volumen. El método consiste en subdividir el volumen en tetraedros, que se forman uniendo cada triángulo del modelo con un punto arbitrario (habitualmente el centro de masas). Posteriormente, se calcula iterativamente la posición del centro de masas y el tensor de inercia de cada tetraedro y se van sumando sucesivamente de forma adecuada. 


\subsection{Extracción de parámetros cinemáticos y dinámicos}

Una vez establecido el método para obtener las coordenadas terreno del bloque, como sabemos que el intervalo de tiempo entre dos fotogramas consecutivos es de $2.5 \mathrm{~ms}$, podremos calcular las velocidades a lo largo de la trayectoria. A estas velocidades de muestreo, incluso en los tramos más rápidos de la trayectoria, somos capaces de parar el bloque y decidir con precisión el instante anterior y posterior de un impacto. Con esta información seremos capaces de medir las velocidades de entrada y de salida y, a partir de aquí, podremos estimar los coeficientes de restitución para el punto de impacto (Asteriou et. al. 2012). Por otra parte, disponemos de las masas de los bloques $y$, en consecuencia, calculamos las energías cinéticas antes y después de los impactos y sabemos qué parte de la energía es disipada $E_{B}+E_{T}$ durante el impacto $\left(E_{B}\right.$ se refiere a la deformación del bloque y $E_{T}$ a la del terreno).

De la misma manera se puede medir la velocidad de rotación del bloque y, por comparación entre los fotogramas instantes antes y después del impacto, podremos medir los cambios que se produzcan en cada interacción con la vertiente e incorporar la energía de rotación del bloque en el balance de energías del sistema.

En la Figura 4 se muestra el balance de energía donde $E_{i}$ se refiere a la energía de impacto como la suma de la energía cinética y energía de rotación. Con $E_{r}$ notamos la energía de rebote que, a su vez, será la suma de las energías cinética y de rotación después del impacto. Cuando el impacto se produce con fragmentación el cálculo se hace más complicado puesto que no disponemos de los modelos 3D de los fragmentos. Por lo tanto, quedaría por hacer una estimación aproximada de sus masas o bien hacer una reconstrucción tridimensional a partir de las imágenes de los tres vídeos. Esta última opción es complicada porque la resolución es demasiado baja y dependemos de cómo giran los fragmentos en el espacio para determinar su forma.
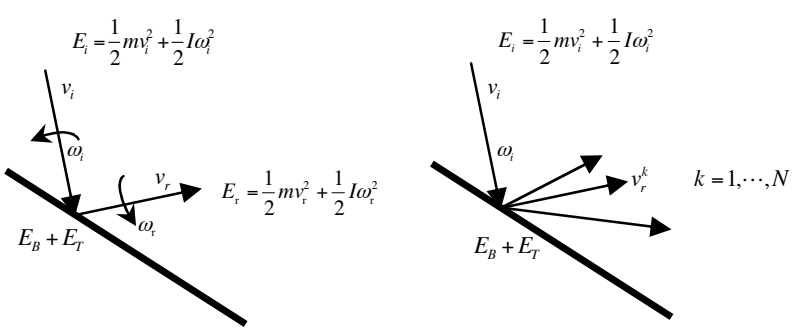

Figura 4: Intercambio de energías en los casos en que no se produzca fragmentación (izquierda) y en los que sí se produce (derecha). En este último, sólo si se pudiera estimar la masa de los fragmentos seríamos capaces de calcular el balance energético.

Por otra parte, podemos estimar cuales son los coeficientes de restitución que dependen de las velocidades que acabamos de medir. En este caso hemos de descomponer las componentes normal y tangencial, $R_{n}$ y $R_{t}$ respectivamente, según el ángulo de incidencia respecto de la normal local de la superficie en este punto. Las expresiones utilizadas con más frecuencia (en la literatura se pueden encontrar otras formas que incluyen, por ejemplo, las velocidades al cuadrado) son, Ec. (2):

$$
R_{n}=\frac{v_{r n}}{v_{i n}}, R_{t}=\frac{v_{r t}}{v_{i t}}
$$

donde $v_{r n}$ es la velocidad de entrada normal, $v_{i n}$ es la velocidad de rebote normal, $v_{i t}$ es la velocidad de entrada tangencial y, por último, $v_{r t}$ es la velocidad de rebote tangencial.

En el momento de escribir este texto ya se han procesado los bloques de forma preliminar. Falta refinar algunos de los procesos para mejorar la precisión en las medidas, pero aún así se muestran algunos de los primeros resultados.

En la Figuras 5 y 6 se muestran las gráficas de las componentes (Easting $v_{x}$, Northing $v_{y}$, y cota ortométrica $v_{z}$ ) de la velocidad respecto del tiempo. Se puede apreciar en ellas los cambios de velocidad que se producen en los momentos en los que el bloque interacciona con la vertiente. En la gráfica inferior, la de la velocidad $v_{z}$, se aprecia que, aproximadamente, en el instante $t=2.8 \mathrm{~s}$ se produce un cambio muy brusco de velocidad que corresponde al instante en que el bloque choca contra el suelo. A partir de ese momento se alternan velocidades positivas, de las caídas, y velocidades negativas, de los rebotes hasta que, finalmente, el bloque se para. En la misma gráfica, pero en el intervalo entre $t=1.5 \mathrm{~s}$ y $t=2.5 \mathrm{~s}$ se aprecia una porción de recta con una pendiente igual a la gravedad. Esta porción corresponde a un intervalo de tiempo en que el bloque estaba en caída libre. Sería de esperar que las velocidades perpendiculares a la línea de la plomada fueran constantes y que únicamente en la dirección de la plomada debiera actuar la aceleración de la gravedad. Las variaciones de velocidad no esperadas que se aprecian en las gráficas son debidas a los errores en las medidas y sobretodo porque en esta versión del programa se mide sobre la superficie del bloque (y no sobre el centro de masas). Además, dado que el bloque gira durante la caída, se debe cambiar de punto de medida cada cierto tiempo buscando el centro del bloque de forma aproximada. Estos dos efectos combinados hacen que las medidas de posición tengan cierta incertidumbre (estimamos unos $\pm 10 \mathrm{~cm}$ ) que se propaga al cálculo de la velocidad y demás magnitudes derivadas. Para evitar estos efectos, se está implementado un módulo que asista al usuario indicando la proyección del centro de masas del bloque vista desde la cámara, como ya se apuntaba en el apartado 4.3.

\section{Propagación de los errores}

Junto a los resultados preliminares hemos obtenido una estimación de los errores asociados al cálculo de la posición, la velocidad y la aceleración.

Como hemos mencionado en el apartado 4.4, el tramo de trayectoria en el intervalo de tiempo en estudio corresponde a la caída libre de un bloque entre dos impactos contra el talud. Sabemos que su centro de masas describe una parábola $y$, por lo tanto, hacemos un ajuste de mínimos cuadrados (Björck 1996) de las observaciones, la cota respecto del tiempo, a un polinomio de segundo grado, Ec. (3): 


$$
z=a_{0}+a_{1} t+a_{2} t^{2}
$$

Donde $a_{0}$ es la cota inicial, $a_{1}$ es la velocidad inicial y $a_{2}$ corresponde a la aceleración $-2 g$. Los errores estimados se han calculado a partir de matraiz cofactor y la desvianción tipo a posteriori y quedan reflejados en la Tabla 1.

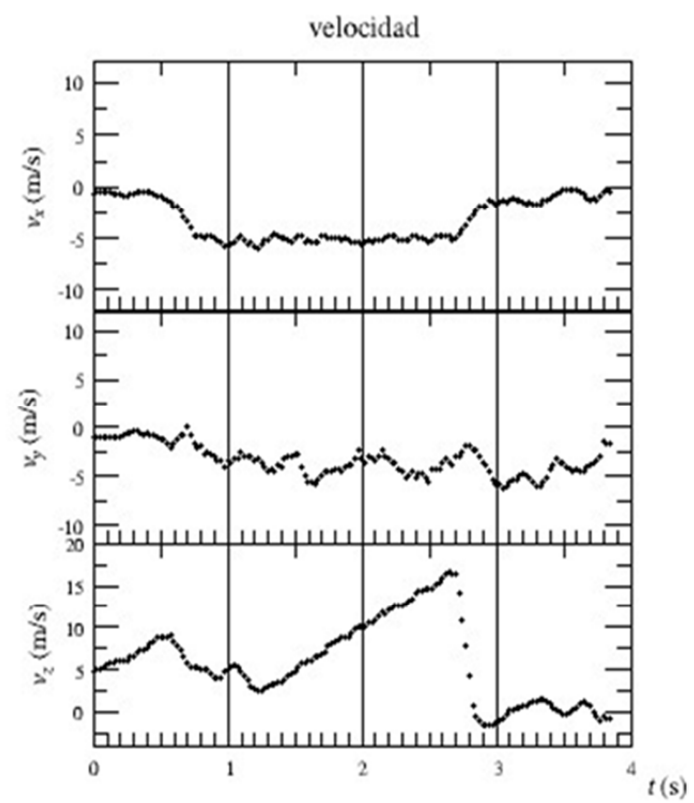

Figura 5: Velocidades respecto del tiempo, componentes de arriba a abajo: este, norte y cota ortométrica.

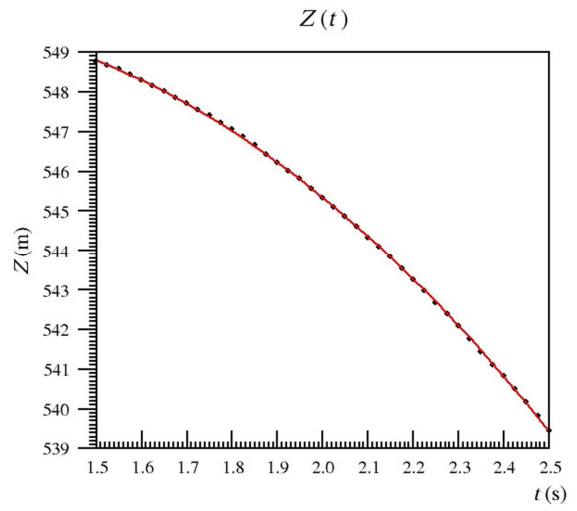

Figura 6: Porción de la Figura 5 ampliada sobre la zona en caída libre del bloque. En rojo se indica la parábola ajustada a los puntos observados.
Tabla 1: Valores resultantes del ajuste de una parábola para los valores de la cota respecto del tiempo, y sus errores.

\begin{tabular}{c|c|c} 
Parámetros & Valor & Error \\
\hline$a_{0}$ & $544.5 \mathrm{~m}$ & $\pm 0.1 \mathrm{~m}$ \\
$a_{1}$ & $10.2 \mathrm{~m} / \mathrm{s}$ & $\pm 0.1 \mathrm{~m} / \mathrm{s}$ \\
$a_{2}$ & $-4.89 \mathrm{~m} / \mathrm{s} 2$ & $\pm 0.03 \mathrm{~m} / \mathrm{s} 2$
\end{tabular}

Los dos primeros parámetros $a_{0}$ y $a_{1}$ dependen de la posición donde impacta el bloque contra la ladera y la velocidad con que rebota y no aportan nada más. Sin embargo, el parámetro $a_{2}$ está ligado a la aceleración de la gravedad que es un valor conocido $g=9.808 \mathrm{~m} / \mathrm{s}^{2}$. Por lo tanto, comparando este valor con el valor estimado por nosotros $\hat{g}=9.78 \pm 0.06 \mathrm{~m} / \mathrm{s}^{2}$ vemos que es compatible y, por lo tanto, dentro de los márgenes de error, queda validado todo el procedimiento descrito en este trabajo.

\section{Conclusiones}

En este trabajo se muestra que un levantamiento topográfico como apoyo a la fotogrametría es imprescindible para obtener coordenadas de alta precisión. Con la base de tiempo que da la electrónica de las cámaras de vídeo de alta velocidad se pueden calcular velocidades y aceleraciones precisas en cada punto de la trayectoria de un bloque. Por otra parte, si se dispone del modelo tridimensional del bloque se pueden estimar su volumen, su masa y el tensor de inercia y, a partir de aquí, la energía cinética antes y después de un impacto. De las velocidades podemos extraer los coeficientes de restitución y los balances de energía.

En el futuro inmediato se harán mejoras para medir directamente la posición del centro de masas y evitar los errores que se producen al medir sobre la superficie del bloque. También se profundizará en el análisis de la propagación de errores desde las posiciones a las velocidades y aceleraciones.

\section{Agradecimientos}

Este trabajo se enmarca dentro del proyecto de investigación titulado "Caracterización y modelización de los desprendimientos rocosos", con acrónimo Rockmodels financiado por el Ministerio de Economía, Industria y Competitividad (BIA2016-75668-P).

\section{References}

ASTERIOU, P., SARAGLOU, H. and TSIAMBAOS, G., 2012. Geotechnical and kinematic parameters affecting the coefficient of restitution for rockfall analysis. Interernational J. of Rock Mechanics and Mining Sciences, 5, pp. 103113.

BJÖRCK, ÅKE, 1996. Numerical Methods for Least Squares Problems. Ed. SIAM.

BLOW, J. and ATMAN J BINSTOCK, 2004. How to find the inertia tensor (or other mass properties) of a 3D solid body represented by a triangle mesh. Internal document at: http:// number-none.com/blow/inertia/bb_inertia.doc [2017].

CHAU, K.T., WONG, R.H.C and WUB, J.J., 2002. Coefficient of restitution and rotational motions of rockfall impacts. Int.J.of Rock Mech. \& Mining Sciences. Vol.39, pp. 69-77. 
COROMINAS, J., LANTADA, N., GILI, J., RUIZ-CARULLA, R., MATAS, G., MAVROULI, O., NÚÑEZ-ANDRÉS, M. ,MOYA, J., BUILL, F., ABELLÁN, A., PUIG, C., PRADES, A., MARTÍNEZ-BOFILL, J. and SALÓ, L., 2017. THE ROCKRISK PROJECT: ROCKFALL risk quantification and prevention, in 6th Interdisciplinary Workshop on Rockfall Protection RocExs 2017.

COROMINAS J. and MAVROULI O., 2011. Rockfall Quantitative Risk Assessment, Chapter 8. In: Lambert, S. and F. Nicot (Eds.): Rockfall engineering. ISTE Ltd \& John Wiley and Sons, Inc. pp. 255-301.

COROMINAS J., MAVROULLI O. and MOYA J., 2013. Metodología para la Evaluación Cuantitativa del Riesgo por desprendimientos de rocas en escala específica y local. VIII Simposio Nacional sobre Taludes y Laderas Inestables.

CROSTA, G.B. and AGLIARDI, F., 2003. A new methodology for physically based rockfall hazard assessment. Natural Hazards and Earth System Sciences 3, pp. 407-422.

DORREN, L.K.A., 2010. Rockyfor3D revealed - description of the complete 3D rockfall model, Tech. Rep., EcorisQ, http://www.ecorisq.org.

GIACOMINI, A., BUZZI, O., RENARD, B. and GIANI, G.P., 2009. Experimental studies on fragmentation of rock falls on impact with rock surfaces. International Journal of Rock Mechanics and Mining Sciences, 46(4), pp. 708-715.

GIACOMINI, A., THOENI, K., LAMBERT, C., BOOTH, S. and SLOAN, S., 2012. Experimental study on rockfall drapery systems for open pit highwalls, International Journal of Rock Mechanics and Mining Sciences. Vol 56, pp. 171-181.

GILI, J.A., RUIZ-CARULLA, R., MATAS, G., COROMINAS, J., LANTADA, N.; NÚÑEZ, M.A., MAVROULI, O., BUILL, F., MOYA, J., PRADES, A. and MORENO, S., 2016. Experimental study on rockfall fragmentation: in situ test design and firsts results. XII International Symposium on Landslides. Naples, Italy.

HARTLEY, R. and ZISSERMAN, A., 2008. Multiple View Geometry. Cambridge University Press.

JONES, C.L., HIGGINS, J.D. and ANDREW, R.D., 2000. Colorado Rock Fall Simulation Program Version 4.0. Colorado Department of Transportation, Colorado Geological Survey.

LI, L. and LAN, H., 2015. Probabilistic modeling of rockfall trajectories: a review, Bulletin of Engineering Geology and the Environment, Volume 21: 1-14.

MATAS, G., LANTADA, N., COROMINAS, J., GILI, J.A., RUIZ-CARULLA, R. and PRADES, A., 2017a. Procedure for assessing the performance of a rockfall fragmentation model. Geophysical Research Abstracts, Vol. 19, EGU201717709, 2017. EGU General Assembly 2017, Vienna (Austria).

MATAS, G., LANTADA, N., COROMINAS, J., GILI, J.A., RUIZ-CARULlA, R. and PRADES, A., 2017b. RockGis application to rockfall. Extended abstract previsto enviar a RocExs, Mayo 2017, Barcelona (España).

PRESS, W.H., TEUKOLSKY, S.A., VETTERLING, W.T., and FLANNERY, B.P., 2007. Numerical Recipes: The Art of Scientific Computing. Third edition. Cambridge University Press.

SALCIARINI, D., TAMAGNINI, C. and CONVERSINI, P., 2009. Numerical approaches for rockfall analysis: a comparison, Pro-ceedings 18th World IMACS / MODSIM Congress, Cairns, Australia.

STEVENS, W.D., 1998. RocFall: a Tool for Probabilistic Analysis, Design of Remedial Measures and Prediction of Rockfalls. A thesis submitted in conformity with the requirements for the degree of Master of Applied Science, Graduate Department of Civil Engineering, University of Toronto.

VOLKWEIN, A., SCHELLENBERG, K., LABIOUSE, V., AGLIARDI, F., BERGER, F., BOURRIER, F., DORREN, L.K.A., GERBER, W. and JABOYEDOFF, M., 2011. Rockfall characterisation and structural protection - a review. Natural Hazards and Earth System Science, 11(9).

WANG, Y. and TONON, F., 2011. Discrete Element Modeling of Rock Fragmentation upon Impact in Rock Fall Analysis, Rock Mechanics and Rock Engineering 44, pp: 23-35.

ZHANG ZX, KOU SQ, JIANG LG and LINDQVIST PA., 2000. Effects of loading rate on rock fracture: fracture characteristics and energy partitioning. Int J Rock Mech Min Sci 37, pp.745-762. 